\title{
EFEITO DO SISTEMA ORGÂNICO ÁGUA-ACETONA E DA TÉCNICA SALTING-OUT NA DESIDRATAÇÃO DA FRUTOSE A 5- HIDROXIMETILFURFURAL
}

\author{
FILIPE N. D. C. GOMES ${ }^{1}$, YGOR T. SOUZA ${ }^{1}$, NIELSON F. P. RIBEIROํ e MARIANA M. V. M. \\ SOUZA $^{1}$ \\ ${ }^{1}$ Universidade Federal do Rio de Janeiro, Escola de Química. \\ E-mail para contato: mmattos@eq.ufrj.br
}

\begin{abstract}
RESUMO - O 5-hidroximetilfurfural (HMF), um importante intermediário em biorefinarias, é obtido através da desidratação de carboidratos. Contudo, sua síntese ainda não é bem consolidada. Assim, este estudo visa analisar o efeito do sistema orgânico e da técnica salting-out nesse processo. A desidratação da frutose foi realizada em reator batelada sob pressão autógena, na presença de $\mathrm{H}_{3} \mathrm{PO}_{4}(1 \% \mathrm{~m} / \mathrm{m})$. A concentração de substrato, temperatura e a agitação foram fixadas em 125 g.L. $\mathrm{L}^{-1}, 180^{\circ} \mathrm{C}$ e $450 \mathrm{rpm}$, respectivamente. Variou-se a proporção volumétrica entre as fases orgânica e aquosa (1:1 e 1:2), o tempo reacional (3 a $30 \mathrm{mim}$ ) e a presença de $\mathrm{NaCl}$ no sistema reacional. Os ensaios revelaram que a utilização do sistema orgânico trouxe uma diminuição considerável das reações paralelas, quando comparado ao sistema aquoso, e que o rendimento em HMF apresenta um máximo entre 5 e $10 \mathrm{~min}$. Os resultados de rendimento nos sistemas orgânicos e bifásicos foram bastante semelhantes, ficando na faixa de 75 a $80 \%$, valor superior a reação em meio aquoso. $\mathrm{O}$ uso de $\mathrm{NaCl}$ provocou uma redução no tempo reacional para se chegar a esse valor de rendimento e o aumento da proporção de co-solvente trouxe uma maior extração de HMF para a fase orgânica no sistema bifásico.
\end{abstract}

\section{INTRODUÇÃO}

A biomassa constitui uma matriz extremamente rica e variada de compostos produzidos pela atividade biológica natural, sendo a fonte de substratos para as biorrefinarias. Estas unidades tecnológicas são fundamentais para a crescente demanda energética e de produtos químicos, pois podem substituir a forte dependência por recursos fósseis, que chega a mais de $90 \%$ na indústria química de compostos orgânicos (Strasser e Vuyyuru, 2012). Contudo, as tecnologias associadas às biorrefinarias ainda não se desenvolveram por completo, devido à limitada disponibilidade de processos de conversão da biomassa em moléculas importantes para o desenvolvimento de plataformas químicas de base renovável. Neste contexto encontra-se o 5-hidroximetilfurfural (HMF), um derivado furânico bifuncional (é concomitantemente um álcool primário e um aldeído aromático), capaz de originar uma vasta gama de compostos químicos vistos como futuros substitutos dos derivados petroquímicos, como por exemplo, o FDCA (ácido 2,5-dimetilfurânico), tido como substituto direto do ácido tereftálico, e o DMF (2,5-dimetilfurano), um biocombustível com potencial para uso em veículos convencionais. 
A rota mais viável para a síntese do HMF é a desidratação da frutose, na presença de catalisadores ácidos, pois sua conformação furanose se assemelha bastante a estrutura química do HMF, como pode ser visto na Figura 1 (Lewkowski, 2001; Kamm et al., 2010). Outros carboidratos (glicose e sacarose, por exemplo) também podem ser utilizados como matéria prima para a síntese desse composto, contudo, devido a sua conformação estrutural, os rendimentos para HMF são bem inferiores quando comparados a utilização da frutose (Moreau et al. 2006; Takeuchi et al., 2007). Porém, o uso da frutose não garante elevados rendimentos devido à grande formação de subprodutos (furfural, huminas e polímeros solúveis) e a reidratação do HMF aos ácidos levulínico e fórmico, pois as condições reacionais também são favoráveis a esses compostos. Neste sentido, vários trabalhos estão sendo realizados para tentar ultrapassar estas barreiras que impedem a produção em larga escala do HMF, principalmente, na busca por catalisadores mais seletivos e meios reacionais menos favoráveis a reidratação.

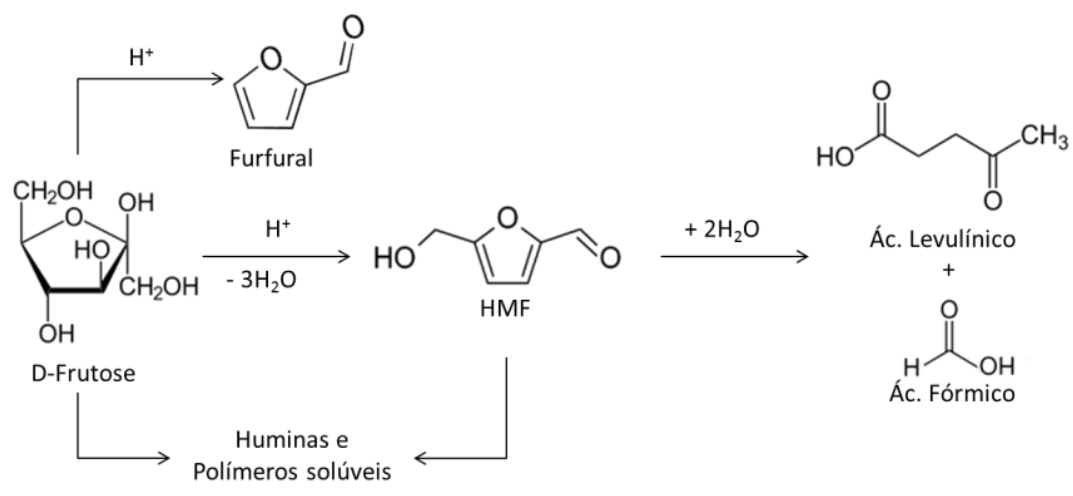

Figura 1 - Reação de desidratação da frutose e reações paralelas.

Vários catalisadores homogêneos e heterogêneos já foram utilizados na síntese do HMF, como por exemplo: nióbia fosfatada (Carlini et al., 1999); resinas ácidas e zeólitas (Shimizu et al., 2009); ácidos minerais - $\mathrm{H}_{3} \mathrm{PO}_{4}, \mathrm{H}_{2} \mathrm{SO}_{4}, \mathrm{HCl}$ - (Takeuchi, 2007; Román-Leshkov et al., 2006; Hansen et al., 2009; Román-Leshkov e Dumesic, 2009). Contudo, vários autores relataram significante desativação dos catalisadores heterogêneos após horas de reação pela deposição de coque e polímeros insolúveis sobre a superfície catalítica. Estudos recentes reportaram com sucesso a utilização de líquidos iônicos como meio reacional para essa síntese, uma vez que os polímeros naturais são solúveis nestes meios e os mesmos atuam como catalisadores da reação, além de serem reutilizados no processo e evitarem a reidratação do HMF. No entanto, os líquidos iônicos apresentam um custo de aplicação muito elevado (Hu et al., 2013).

O uso de solventes orgânicos também é favorável à síntese do HMF, pois a ausência de água impede sua reidratação, porém a solubilidade dos açucares e do HMF nestes solventes é bastante baixa e a separação do produto final é complexa. A literatura reporta que a utilização de co- 
solventes orgânicos para a formação de sistemas bifásicos eleva o rendimento do HMF, pois o mesmo é removido continuamente para a fase orgânica, deslocando o equilíbrio reacional em favor da produção de HMF, o que pode ser otimizado pelo uso da técnica salting out. Román-Leshkov e Dumesic (2009) reportaram diversos experimentos para esta técnica e concluíram que cloretos monovalentes proporcionaram um aumento da extração do HMF da fase orgânica. Além disso, os autores concluíram que solventes com quatro carbonos em sua composição geraram rendimentos para HMF mais elevados, porém solventes com três carbonos também demostraram ser apropriados para a síntese de HMF, uma vez que a acetona apresentou resultados semelhantes ao 2-butanol, que apresentou o melhor rendimento.

O presente trabalho relata uma nova abordagem para a síntese do HMF a partir da desidratação da frutose, através de um estudo mais aprofundado da influência do meio reacional, com foco na utilização da acetona como co-solvente e o uso da técnica 'salting out'.

\section{EXPERIMENTAL}

As reações de desidratação da frutose a HMF foram realizadas em meio homogêneo utilizando o ácido (orto)fosfórico, $\mathrm{H}_{3} \mathrm{PO}_{4}$, como catalisador. Estes ensaios foram conduzidos em reator batelada, com capacidade de $300 \mathrm{~mL}$, sob pressão autógena a uma temperatura de $180{ }^{\circ} \mathrm{C}$ e agitação de 450 rpm. As concentrações de substrato e catalisador foram fixadas em 125 g.. $\mathrm{L}^{-1}$ e $1 \% \mathrm{~m} / \mathrm{m}$, respectivamente. Variou-se a proporção volumétrica entre as fases orgânica e aquosa (1:1 e 1:2), com um volume total de $200 \mathrm{~mL}$, bem como o tempo reacional (3 a $30 \mathrm{mim}$ ). Para os ensaios com a presença de cloreto de sódio $(\mathrm{NaCl}$ - 300 g.L.-1 $)$, a variação da proporção volumétrica entre as fases orgânica e aquosa foi mantida em 1:1 e 1:2 e o tempo reacional foi variado de 3 a 15 minutos. Para efeito de comparação, foi realizado um ensaio com as mesmas condições reacionais, porém tendo apenas água como solvente.

As amostras reacionais foram analisadas por cromatografia líquida de alta eficiência (HPLC), utilizando equipamento Shimadzu equipado com uma coluna Aminex HPX-87H (Biorad) e detectores de índice de refração e UV-vis ligados em série. Neste, o comprimento de onda de absorção medido foi de $210 \mathrm{~nm}$. Uma solução de $\mathrm{H}_{2} \mathrm{SO}_{4}$ a $0,01 \mathrm{M}$ foi utilizada como fase móvel, com um fluxo de 0,8 mL.min ${ }^{-1}$ e temperatura da coluna de $55{ }^{\circ} \mathrm{C}$. A conversão de frutose $\left(\mathrm{C}_{\mathrm{F}}\right)$, o rendimento a $\mathrm{HMF}$ $\left(\mathrm{R}_{\mathrm{HMF}}\right)$ e o coeficiente de partição são descritos nas equações (1-3), respectivamente.

$$
\begin{aligned}
& C_{F}=\frac{\text { Moles de frutose reagios }}{\text { Moles de frutose iniciais }} \times 100 \\
& R_{H M F}=\frac{\text { Moles de HMF produzidos }}{\text { Moles de frutose iniciais }} \cdot 100
\end{aligned}
$$




$$
R=\frac{\text { Concentração mássica de HMF na fase orgânica }}{\text { Concentração mássica de HMF na fase aquosa }}
$$

\section{RESULTADOS E DISCUSSÃO}

Primeiramente, é importante ressaltar que as condições reacionais usadas neste estudo (temperatura, concentração de substrato e catalisador) foram otimizadas em pesquisas anteriores do grupo e que em todas as reações de desidratação de açúcares ocorre o escurecimento da solução final devido a reações de caramelização que ocorrem concomitantemente à desidratação. Em todos os ensaios de síntese de HMF aqui explanados, os mesmos apresentaram a cor marrom claro ou escuro dependendo do teor de polímeros solúveis e huminas.

De acordo com os ensaios utilizando o meio orgânico (sistema água/acetona), cujos resultados estão apresentados na Figura 2, a conversão de frutose apresentou-se praticamente constante, em torno de $95 \%$, desde o início da reação (3 min) - o mesmo comportamento também foi observado para a reação em meio aquoso. Já o rendimento para o HMF apresentou um comportamento diferente entre os meios reacionais utilizados. Em meio aquoso, o rendimento apresentou-se constante a $55 \%$

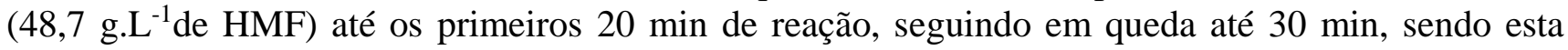
queda associada à grande presença de huminas no meio reacional (as huminas não puderam ser quantificadas por HPLC ou outra técnica analítica). Como exposto anteriormente, as reações de caramelização são as responsáveis pela formação desses compostos. Basicamente, essas reações são um complexo conjunto de desidratações e hidrólises que passam pela formação de anidridos ou de anéis insaturados (ligações duplas), responsáveis pela cor nos sistemas reacionais. Ao final do processo tem-se a predominância de ácidos orgânicos, aldeídos e grupos enólicos (Oetterer, 2008). Resultado similar de seletividade foi obtido por Roman-Leshkov e seus colaboradores (2006), cerca de $51 \%$, porém com baixa conversão, apenas $50 \%$, mesmo em condições operacionais análogas, diferenciando apenas a concentração de frutose de $300 \mathrm{gL}^{-1}$ e $0,25 \mathrm{M} \mathrm{HCl}$. Takeuchi et al. (2007) também relataram rendimentos análogos, porém com o uso de glicose.
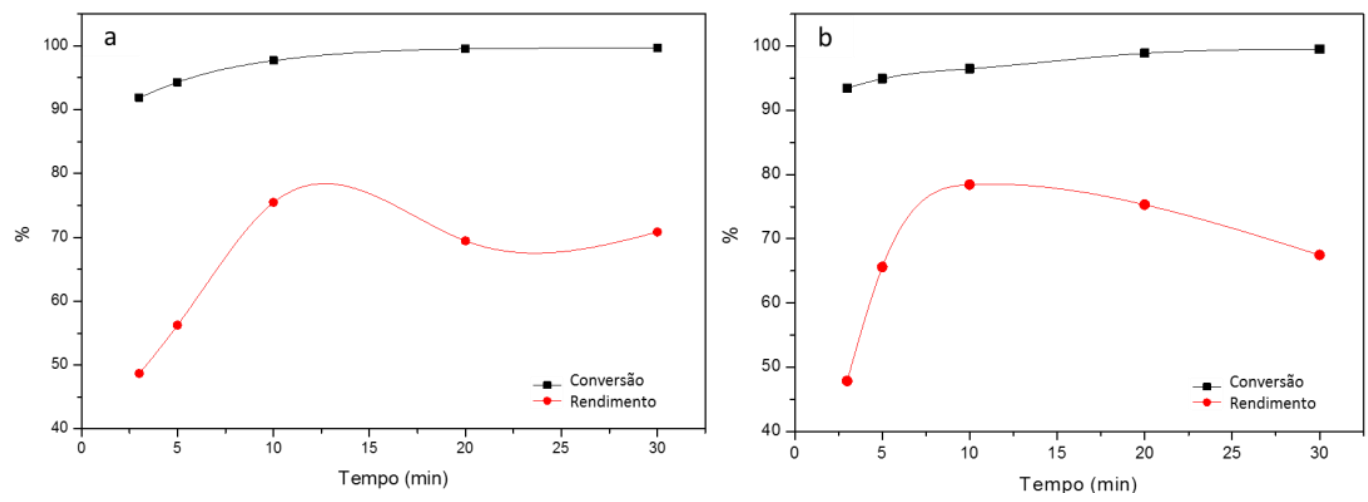

Figura 2 - Efeito do tempo reacional na síntese do HMF utilizando sistema água:acetona.

$$
\text { (a: Água:Acetona = 1:1; b: Água:Acetona = 1:2). }
$$




\section{9 a 22 de outubro de 2014 \\ Florianópolis/SC}

Em meio orgânico, o rendimento para HMF passa por um máximo em torno de 10 min, atingindo um valor de $75 \%$ (66,0 g.L $\mathrm{L}^{-1}$ de HMF) para os dois sistemas avaliados neste estudo (água:acetona $=1: 1$ e 1:2). Isto indica que, para o sistema água/acetona, o teor do co-solvente não interfere no rendimento para $\mathrm{HMF}$, pois o meio reacional em questão é homogêneo e assim o HMF continua em solução aquosa, passível de reidratação e polimerização - reações responsáveis pelo decaimento do rendimento com o decorrer do tempo reacional. Contudo, a adição de um co-solvente trouxe uma elevação do rendimento para HMF em comparação a reação conduzida em meio aquoso sob as mesmas condições reacionais, elevando o rendimento de $55 \%$ para $75 \%$.

A Figura 3 apresenta o rendimento a HMF em função do tempo para os ensaios em que foi utilizada a técnica do salting out. Esta técnica consiste na adição de um sal inorgânico no sistema reacional a fim de saturar a fase aquosa e assim promover a separação entre as fases, criando um sistema bifásico com solventes que são completamente miscíveis na ausência de sal. A concentração de cloreto de sódio utilizada foi de 300 g... $\mathrm{L}^{-1}$, valor correspondente ao ponto de saturação da fase aquosa. O benefício da utilização do sistema bifásico consiste na extração contínua do HMF formado na fase aquosa para a fase orgânica. Assim sendo, o tempo de residência do HMF em água é diminuído e as reações secundárias são, por conseguinte, minimizadas (Raman-Leshkov et al., 2006; Chheda et al., 2007; Kamm et al., 2010). Vale salientar que a conversão de frutose não pode ser quantificada nesses ensaios devido à presença de interferências nas análises de HPLC até 15 min de reação, após este tempo a conversão, em ambos os ensaios, foi de $100 \%$. Além disso, os intermediários de reação - referentes a cada estágio da desidratação da frutose a HMF - não puderam ser quantificados por HPLC, pois sua formação e seu consumo ocorrem rapidamente.

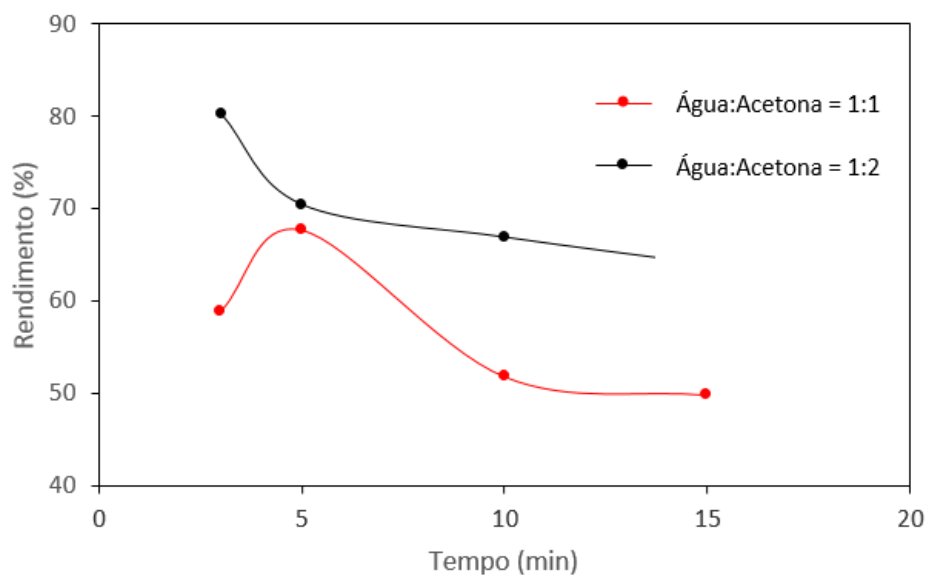

Figura 3 - Rendimento em HMF utilizando a técnica salting out em sistema água:acetona.

De acordo com a Figura 3, nota-se que a adição de sal no sistema orgânico água/acetona originou resultados distintos para cada proporção de co-solvente utilizada, quando comparado aos seus respectivos ensaios na ausência de sal (Figura 2). A presença de sal no sistema com proporção água:acetona de 1:1 não provocou o efeito esperado, ou seja, o rendimento para HMF não sofreu nenhuma alteração considerável quando comparado ao ensaio nas mesmas condições, mas sem a presença de sal (Figura 2a). Em ambos os ensaios o rendimento a HMF ficou na faixa de 70 a $75 \%$. De acordo com as Figuras 2 e 3, pode-se perceber que o comportamento para ambos os ensaios é bem 


\section{9 a 22 de outubro de 2014 \\ Florianópolis/SC}

similar, passando por um ponto máximo de rendimento, porém em um menor tempo, mostrando que o $\mathrm{NaCl}$ também atua como um promotor reacional tanto para a etapa de desidratação quanto para as reações paralelas, pois o decaimento do rendimento com o tempo é superior, quando comparado ao ensaio correspondente sem sal. O mesmo também foi relatado por Liu et al. (2012) em seu estudo sobre promotores inorgânicos na síntese de HMF.

Para a proporção água:acetona de 1:2, o $\mathrm{NaCl}$ afetou positivamente o rendimento quando comparado aos demais ensaios. A ação deste sal como promotor reduziu o tempo reacional no qual o rendimento máximo para HMF foi alcançado, além de elevar o rendimento para o mesmo quando comparado ao ensaio com a proporção água:acetona de 1:1. Resultados similares também foram observados por Román-Leshkov e Dumesic (2009); contudo, a conversão da frutose não foi total, apenas $62 \%$. As condições reacionais foram semelhantes, $30 \% \mathrm{~m} / \mathrm{m}$ de frutose, $\mathrm{pH}$ 0,6 (usando $\mathrm{HCl}$ ), temperatura de $150{ }^{\circ} \mathrm{C}$ por $35 \mathrm{~min}$, com razão entre as fases de 3,2. Deste modo, é visto que uma melhor conversão da frutose é alcançada com temperaturas superiores a $150{ }^{\circ} \mathrm{C}$ e menores tempos reacionais. Outro aspecto importante é o fato de que os autores obtiveram esse resultado com uma maior quantidade de fase orgânica e mesmo assim o coeficiente de partição do HMF $(R=3,6)$ foi equivalente ao observado neste estudo para a proporção entre as fases de $1: 2$, com $\mathrm{R}=3,4$ e superior para a proporção entre as fases de $1: 1$, com $\mathrm{R}=3,0$. Isto indica que a partir da proporção entre as fases de 1:2, o efeito da adição de mais fase orgânica não ocasiona uma melhor partição do HMF. Ressalta-se que o coeficiente de partição $(\mathrm{R})$ foi calculado após a reação e este valor é referente ao ponto de melhor rendimento para HMF em cada ensaio.

\section{CONCLUSÕES}

A avaliação da influência do meio reacional, utilizando o sistema água/acetona, revelou que o rendimento a HMF é afetado negativamente pela presença de água no sistema reacional, uma vez que o meio aquoso favorece as reações de condensação e reidratação. Com uso do sistema orgânico o rendimento em HMF foi significativamente maior do que em meio aquoso, passando de $55 \%$ para $75 \%$. Porém, para este sistema, o aumento do teor de co-solvente não apresenta efeito sobre o rendimento a HMF. Considerando o sistema bifásico (água/acetona e $\mathrm{NaCl}$ ), a presença de sal influenciou positivamente o rendimento a $\mathrm{HMF}$, reduzindo o tempo reacional no qual o rendimento máximo foi alcançado de 10 min para 3 min no sistema bifásico com proporção entre as fases de 1:2, evidenciando a ação de promotor reacional do $\mathrm{NaCl}$. Em conjunto com esta ação promotora, o aumento do teor de co-solvente no sistema bifásico provocou uma maior partição do HMF entre as fases. 


\section{REFERÊNCIAS}

CARLINI, C.;GIUTTARI, M.; GALLETTI, A.M.R.; SBRANA, G. Selective saccharides dehydration to 5-hydroxymethyl-2-furaldehyde by heterogeneous niobium catalysts. Applied Catalysis A,v.183, p. 295-302, 1999.

CHHEDA, J.N.; ROMAN-LESHKOV, Y.; DUMESIC, J.A.Production of 5-hydroxymethylfurfural and furfural by dehydration of biomass-derived monoand poly-saccharides. Green Chem., v. 9, p. 342-350, 2007.

HANSEN, T. S.; WOODLEY, J. M.; RIISAGER, A. Efficient microwave-assisted synthesis of 5hydroxymethylfurfural from concentrated aqueous fructose. Carbohydrate Research, v. 344, p. 25682572, 2009.

HU, Z.; LIU, B.; ZHANG, Z.; CHEN, L. Conversion of carbohydrates into 5-hydroxymethylfurfural catalyzed by acidic ionic liquids in dimethyl sulfoxide. Industrial Crops and Product, V. 50, P. 264269, 2013.

KAMM, B.; GERHARDT, M; LEIB, S. Thermochemical Conversion of Biomass to Liquid Fuels and Chemicals. RSC Publishing, v. 1, p. 49-56, 2010.

LEWKOWSKI, J., Synthesis, Chemistry and Applications of 5-Hydroxymethyl-furfuraland Its Derivatives. Arkivoc, v. 1, p. 17-54, 2001.

LIU, J.; TANG, Y.; WU, K.; BI, C.; CUI, Q. Conversion of fructose into 5-hydroxymethylfurfural (HMF) and its derivatives promoted by inorganic salt in alcohol. Carbohydrate Research, v. 350, p. 20-24, 2012.

MOREAU C., F. A.; VANOYE, L. Dehydration of fructose and sucrose into 5-hydroxymethylfurfural in the presence of 1-H-3-methyl imidazolium chloride acting both as solvent and catalyst. J. Mol. Catal. A: Chem., v. 253, p. 165-169, 2006.

OETTERER, M. Química de alimentos: Escurecimento não enzimático. 2008. Disponível em: $<$ http://www.esalq.usp.br/departamentos/lan/pdf/Quimica\%20de\%20\%20\%20Escurecimento\%20nao \%20enzimatico.pdf> Acessando em 2014.

ROMAN-LESHKOV, Y.; CHHEDA, J. N.; DUMESIC, J. A. Phase Modifiers Promote Efficient Production of Hydroxymethylfurfural from Fructose. Science, v. 312, p.1933-1937, 2006.

ROMAN-LESHKOV, Y.; DUMESIC, J. A. Solvent Effects on Fructose Dehydration to 5Hydroxymethylfurfural in Biphasic Systems Saturated with Inorganic Salts. Top. Catal, v. 52, p.297303, 2009.

SHIMIZU, K.; UOZUMI, R.; SATSUMA, A. Enhanced production of hydroxymethylfurfural from fructose with solid acid catalysts by simple water removal methods. Catal. Comm., v. 10, p. 1849- 
$1853,2009$.

STRASSER, P., VUYYURU, K.R. Oxidation of biomass derived 5-hydroxymethylfurfural using heterogeneous and electrochemical catalysis. Catal. Today, v. 15, p. 144-154, 2012.

TAKEUCHI, Y.; JIN, F.; TOHJI, K.; ENOMOTO, H. Acid catalytic hydrothermal conversion of carbohydrate biomass into useful substances. J. Mater. Sci., v. 43, p. 2472-2475, 2007. 